\title{
Superconductivity in doped cubic silicon: an ab initio study
}

\author{
E. Bourgeois and X. Blase ${ }^{1}$ \\ 1 Laboratoire de Physique de la Matière Condensée et Nanostructures, Université Lyon I; CNRS, \\ UMR 5586, Domaine Scientifique de la Doua, F-69622 Villeurbanne cedex; France.
}

(Dated: July 7, 2018)

\begin{abstract}
We study within a first-principle approach the band structure, vibrational modes and electronphonon coupling in boron, aluminum and phosphorus doped silicon in the diamond phase. Our results provide evidences that the recently discovered superconducting transition in boron doped cubic silicon can be explained within a standard phonon-mediated mechanism. The importance of lattice compression and dopant related stretching modes are emphasized. We find that $\mathrm{T}_{C}$ can be increased by one order of magnitude by adopting aluminum doping instead of boron.
\end{abstract}

PACS numbers: 74.62.Bf, 71.15.Mb, 74.20.Fg, 74.25.Kc

The experimental discovery by Bustarret and coworkers 1] of a superconducting (SC) transition in heavily boron-doped silicon in the diamond phase (labeled $c$-Si in what follows) concluded a long history of research on such a transition in silicon-based systems and in doped semiconductors in general. [2] Until recently, a SC behavior in silicon had only been observed in high-pressure metallic phases such as the hexagonal or $\beta$-tin structures [3] or low-pressure cage-like doped clathrate phases. [4, 5] This latter structure, which becomes superconducting upon heavy doping, bears much similarities with the diamond phase, as it is a semiconducting $s p^{3}$ network, but with a band gap in the visible range. [6]

Besides doped $s p^{3}$ silicon clathrates, superconductivity in $c$-Si was recently made likely by the discovery of a SC transition in highly boron-doped carbon diamond. 7] Tunnelling spectroscopy, [8] reflectivity measurements 9] and first-principles studies within the density functional theory (DFT), performed in the virtual crystal approximation (VCA) 10, 11, 12] or a supercell approach, [13, 14, 15] strongly suggested that the transition was phonon mediated. Further, the study of the SC origin in Si-clathrates and carbon diamond led theorists to predict the SC transition in doped $c$-Si within a crude rigid-band model for doping [5] and a more accurate VCA treatment [10] with emphasis on boron doping.

In this paper, we study by means of $a b$ initio simulations within a supercell approach the electronic, vibrational and electron-phonon coupling properties of boron, aluminum $p$-doped and phosphorus $n$-doped $c$-Si. We find that a standard phonon-mediated BCS approach can account for the experimental transition temperature observed at high boron content. We predict further that aluminum doping would allow to increase $\mathrm{T}_{C}$ by one order of magnitude, an effect ascribed in particular to the negative effect of lattice compression on $\mathrm{T}_{C}$ in the case of boron doping.

The calculations are performed within a planewave pseudopotential implementation [16] of the DFT using the PBE functional [17] for exchange and correlation. Ultrasoft pseudopotentials are used with a 20 Ry (160 Ry) cutoff for the expansion of the wavefunctions (charge density), increased to $25 \mathrm{Ry}$ (200 Ry) in the case of borondoping. A $(2 \times 2 \times 2)$ supercell containing 16 atoms is built with one $\mathrm{Si}$ atom replaced by an impurity, leading to a $\sim 6.25 \%$ doping concentration, in the range of the estimated 5.7-8.4\% experimental value [1] for the superconducting boron-doped samples. The Brillouin zone (BZ) is sampled by a $(5 \times 5 \times 5) \mathrm{k}$-point grid for structural relaxation and the calculation of the phonon modes which are obtained on a $(4 \times 4 \times 4) \mathbf{q}$-grid ( $\mathbf{k}$ and $\mathbf{q}$ will refer to electron and phonon momentum respectively). We adopt a much finer $(10 \times 10 \times 10)$ k-point sampling for calculating the q-dependent electron-phonon coupling constants $\lambda(\mathbf{q})$. A tetrahedron extrapolation technique is used to accelerate the summation over phonon modes in the calculation of the coupling constant $\lambda$.

We first minimize the energy of the system with respect to the cell size and atomic positions. As expected, the largest relaxation occurs for boron doping with an important contraction of the Si-B bonds $(\sim 2.1 \AA)$, leading to a $\sim 1.9 \%$ contraction of unit cell lattice parameter. Experimentally, as doped samples are constrained in the $\widehat{x y}$ plane (parallel to the surface) by the undoped substrate, the relaxation was shown to be anisotropic with a $\epsilon_{z z}$ contraction along the $\hat{z}$-axis ranging from $2.5 \%$ to $3.7 \%$ from XRD analysis. Using a Poisson's ratio $\nu$ of 0.28 , this yields an experimental averaged lattice compression $\epsilon_{a v}=\epsilon_{z z}(1-\nu) /(1+\nu)$ in the $1.4 \%-2.1 \%$ range. Our theoretical value clearly falls within these estimates. This lattice contraction will be shown to have important consequences on $\mathrm{T}_{C}$.

We plot in Fig. 1(a) the band structure of the B:Si cell that we compare to that of undoped silicon at the same lattice parameter (dashed lines). The Fermi level lies 0.55 $\mathrm{eV}$ below the top of the valence bands (VBM), showing that in this limit of large doping, Si:B is a degenerate semiconductor. Clearly, close to the Fermi level, the band structures of doped and pristine silicon are very similar. In the case of $p$-doped aluminum samples, the Fermi level falls $0.45 \mathrm{eV}$ below the VBM (Fig. 1 b).

We now turn to the vibrational properties and plot in 

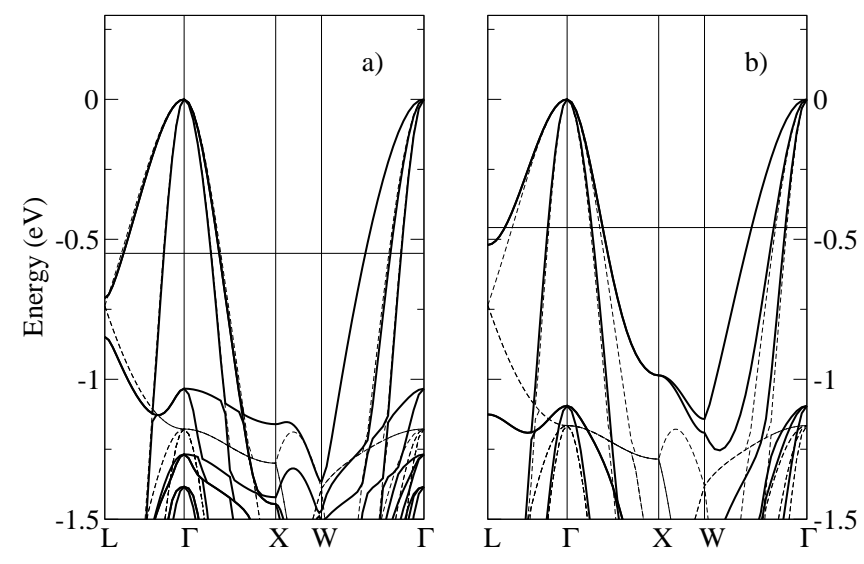

FIG. 1: Electronic DFT band structures of (a) boron-, and (b) aluminum-doped $c$-Si in the $(2 \times 2 \times 2)$ cell. The Fermi levels are indicated by horizontal lines. Dashed lines are for the undoped c-Si.

Fig. 2 the phonon density of states ( $p h$-DOS). [18] As compared to the $501 \mathrm{~cm}^{-1}$ theoretical frequency for the zone-center optical modes in c-Si in the present PBE approach $\left(520 \mathrm{~cm}^{-1}\right.$ experimentally), one observe $\mathrm{a} \sim 40$ $\mathrm{cm}^{-1}$ softening in B-doped and Al-doped samples. In the case of boron-doping, we find that the effect of lattice parameter reduction shifts the zone-center optical modes of pristine $c$-Si to higher frequencies by $20 \mathrm{~cm}^{-1}$. As a result, the effect of $\mathrm{B}$-doping really amounts to a $\sim 60$ $\mathrm{cm}^{-1}$ softening. Further, the strong reduction of the BSi bonds yields a well separated B-Si (3-fold) stretching mode located $\sim 100 \mathrm{~cm}^{-1}$ above the optical modes continuum, a signature in excellent agreement with the Raman peak at 590-600 $\mathrm{cm}^{-1}$ observed experimentally in the superconducting B-doped samples. [1] This mode has been already observed experimentally in non-superconducting samples with a large frequency shift under uniaxial pressure related to the strong local stress around the dopant. [19]

The Eliashberg function $\alpha^{2} F(\omega)$, which allows to analyze which phonons contribute to the electron-phonon coupling constant $\lambda$, is represented in Fig. 2(a-b) (grey thick line) together with the $p h$-DOS (black line). $\alpha^{2} F(\omega)$ can be calculated from the knowledge of the electron-phonon coupling matrix elements, 20] namely:

$$
\begin{aligned}
\alpha^{2} F(\omega) & =N\left(E_{F}\right) \sum_{\mathbf{q} \nu}\left\langle\left|g_{\mathbf{q} \nu}\right|^{2}\right\rangle \delta\left(\omega-\omega_{\mathbf{q} \nu}\right) \\
\left\langle\left|g_{\mathbf{q} \nu}\right|^{2}\right\rangle & =\int \frac{d^{3} \mathbf{k}}{\Omega_{B Z}}\left|g_{\mathbf{q} \nu}^{\mathbf{k} n n^{\prime}}\right|^{2} \frac{\delta\left(\epsilon_{n \mathbf{k}}\right) \delta\left(\epsilon_{n^{\prime} \mathbf{k}+\mathbf{q}}\right)}{N\left(E_{F}\right)^{2}} \\
g_{\mathbf{q} \nu}^{\mathbf{k} n n^{\prime}} & =\left(\frac{\hbar}{2 M \omega_{\mathbf{q} \nu}}\right)^{\frac{1}{2}}\left\langle\psi_{n \mathbf{k}}^{0}\left|\hat{\epsilon}_{\mathbf{q} \nu} \cdot \frac{\delta V^{s c f}}{\delta \hat{u}_{\mathbf{q} \nu}}\right| \psi_{n^{\prime} \mathbf{k}+\mathbf{q}}^{0}\right\rangle(
\end{aligned}
$$

To accelerate the convergence with respect to phonon momentum q-sampling, we observe that the largest vari- ations in $\left\langle\left|g_{\mathbf{q} \nu}\right|^{2}\right\rangle$ comes from the two $\delta$-functions in Eq. 2. By defining the nesting factor $\mathrm{n}(\mathbf{q})$, that can be obtained by setting $g_{\mathbf{q} \nu}$ to unity in Eq. 2, we find that $\left\langle\left|g_{\mathbf{q} \nu}\right|^{2}\right\rangle / n(\mathbf{q})$ is smoothly varying (as a function of $\mathbf{q}$ ) and well behaved at zone-center. From the explicit calculation of such a ratio on the $(4 \times 4 \times 4)$ q-grid, we extrapolate $\left\langle\left|g_{\mathbf{q} \nu}\right|^{2}\right\rangle / n(\mathbf{q})$ using tetrahedron techniques on a much finer (10x10x10)-grid in order to obtained a well converged $\alpha^{2} F(\omega)$ function. 21]

The integration of the Eliashberg function yields the coupling constant $\lambda=2 \int d \omega \alpha^{2} F(\omega) / \omega$ with values of 0.26 and 0.36 for the B-doped and Al-doped systems respectively. 22] Our $\lambda=0.26$ for $\mathrm{B}$-doped $c$-Si is close to the 0.30 value found in Ref. 10 using the VCA for a slightly smaller $5 \%$ doping level. In the present supercell approach, the contribution of the B-related stretching modes at high energy amounts to $15 \%$ of the coupling constant. In the case of Al-doping, there is an enhanced contribution from modes located $\sim 50 \mathrm{~cm}^{-1}$ below the softened optical modes and showing a significant weight onto the $\mathrm{Al}$ displacements. This is reminiscent of the possible importance of the B-related vibrational modes in the case of B:C samples as suggested experimentally [9] and by theory. [13, 14, 15]
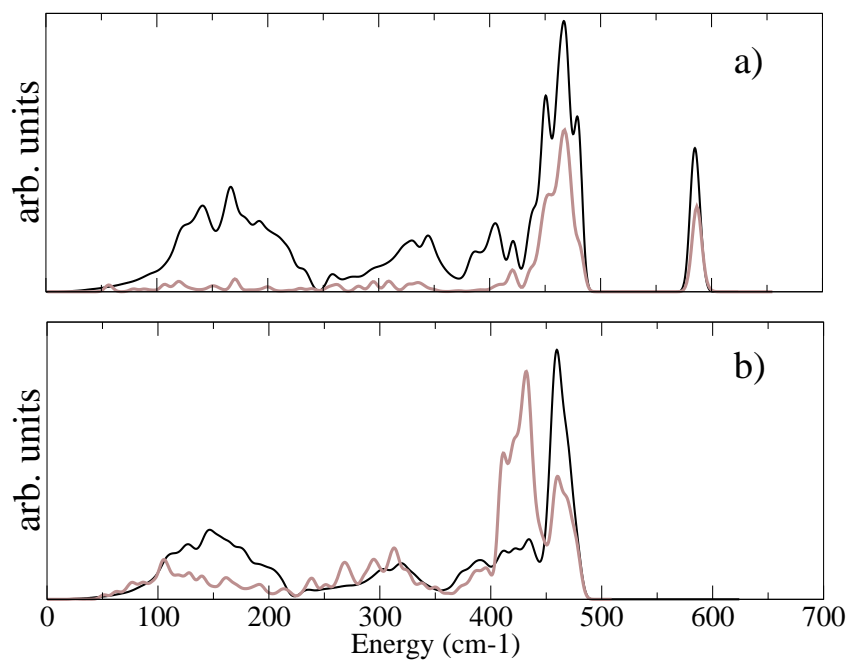

FIG. 2: Phonon-DOS (black line) and Eliashberg functions (grey thick line) for (a) boron-, and (b) aluminum-doped $c$-Si in the $(2 \times 2 \times 2)$ cell.

Using the standard McMillan formula, or a modified relation accounting for the $\delta$-like shape of the Eliashberg function (see Ref. 10), and with an average phonon frequency $\hbar \omega_{0}$ set to the value that maximizes $\alpha^{2} F(\omega)(\sim$ $470 \mathrm{~cm}^{-1}$ ), one obtains for B:Si a transition temperature $\mathrm{T}_{C}$ evolving from $0.24 \mathrm{~K}$ to $0.06 \mathrm{~K}$ for $\mu^{*}$ in the 0.08 0.12 range. Such a temperature is clearly consistent with the set of values obtained in Ref. 1 for the three superconducting samples, with a largest $\mathrm{T}_{C}$ of $0.34 \mathrm{~K}$ for the sample with the highest boron content $(\sim 8.4 \%)$. [1] 
The larger value of $\lambda$ in Al:Si can be partly attributed to a negative pressure effect. In the case of the $\mathrm{Si}$ clathrates, it was shown experimentally and by theory [5] that $\mathrm{T}_{C}$ decreases under applied pressure with a $d T_{C} / d \epsilon \sim 2 \mathrm{~K}$. As a computational experiment, we study $\mathrm{Al}: \mathrm{Si}$ at the B:Si theoretical lattice constant, that is we apply a $2.1 \%$ lattice contraction. We find that $\lambda$ decreases by $\sim 10 \%$, an effect that can be ascribed in particular to a $\sim 6.5 \%$ density of states reduction under broadening of the bands by applied pressure. Further, the expression of $\lambda$ as a function of $\alpha^{2} F(\omega)$, and the inspection of Eqs.(1-3), reveal a $\left(1 / \omega_{\mathbf{q} \nu}\right)^{2}$ dependence of a given phonon mode to the coupling constant. This scaling reduces by a factor $\sim(590 / 470)^{2} \sim 1.58$ the contribution of the three optical bands which, upon introduction of boron, are shifted to a much higher energy, amounting to further reducing $\lambda$ by $0.03-0.04$.

Changes in $\lambda$ do not affect $T_{C}$ directly as the average phonon frequency prefactor $\omega_{0}$ and the Coulomb repulsion parameter $\mu^{*}$ may change as well. The limited evolution of $N\left(E_{F}\right) / \omega_{0}$ suggest that $\mu^{*}$ should hardly change from one type of doping to another. The average frequency $\omega_{0}$ decreases by $\sim 40 \mathrm{~cm}^{-1}$ in the case of aluminum doping. However, this decrease does not overcome the increase in the parameter $\lambda$ and with $\mu^{*}$ in the $0.08-0.12$ range, the transition temperature $\mathrm{T}_{C}$ is found to lie in between $2.8 \mathrm{~K}$ and $0.6 \mathrm{~K}$, that is about one order of magnitude larger than the values obtained in the case of boron doping. Even though the effect of impurity randomness (beyond the supercell approach) [23] and possible anharmonic corrections may change the $\lambda$ values calculated here, we expect this evolution of the coupling constant from boron to aluminum to be robust.

We finally briefly turn to the case of hypothetical highly $n$-doped phosphorus samples. In the $6.5 \%$ doping limit, the system is degenerate, but the conduction bands and the multi-valley structure of pristine c-Si are strongly modified, with in particular a large splitting of the $\Gamma_{15}$ state. We find a $\lambda$ value of 0.30 , not significantly different from the boron case and rather disappointing in view of early arguments in favor of multi-valley semiconductors. 2]

In conclusion, we have studied the structural, electronic and electron-phonon coupling properties in highlydoped c-Si. Our results provide support for a standard phonon-mediated BCS-type mechanism for the occurrence of superconductivity in B-doped silicon. The transition temperature is expected to increase by an order of magnitude upon aluminum doping instead of boron. It is not clear however if the present synthesis techniques (such as the gas immersion laser doping technique used in Ref. 1) may be adapted to other type of dopants and/or to a larger doping percentage. Conflicting phenomena, such as dopant segregation as in the case of boron doped diamond [24] may significantly complicate the search for larger $\mathrm{T}_{C}$ in $c$-Si.

Acknowledgements: Calculations have been performed at the French CNRS national computing center (IDRIS, Orsay). The authors are indebted to the French National Agency for Research (ANR) for funding (contract "SupraDiam" ANR-05-BLAN-0282) and acknowledge E. Bustarret, C. Marcenat, and J. Boulmer for enlightening discussions.

[1] E. Bustarret et al., Nature (London) 444, 465 (2006).

[2] Marvin L. Cohen, Rev. Mod. Phys. 36, 240 (1964); ibid, Phys. Rev. 134, A511 (1964).

[3] K.J. Chang et al., Phys. Rev. Lett. 54, 2375 (1985).

[4] H. Kawaji, H.-o. Horie, S. Yamanaka, and M. Ishikawa, Phys. Rev. Lett. 74, 1427 (1995); K. Tanigaki et al., Nature Mater. 2, 653 (2003).

[5] D. Connétable et al., Phys. Rev. Lett. 91, 247001 (2003).

[6] E. Galvani et al., Phys. Rev. Lett. 77, 3573 (1998); D. Connétable et al., Phys. Rev. Lett. 87, 206405 (2001); X. Blase, Phys. Rev. B 67, 035211 (2003).

[7] E.A. Ekimov et al., Nature 428, 642 (2004); Y. Takano, M. Nagao, I. Sakaguchi, M. Tachiki et al., Appl. Phys. Lett. 85, 2851 (2004); E. Bustarret et al., Phys. Rev. Lett. 93, 237005 (2004).

[8] B. Sacépé et al., Phys. Rev. Lett. 96, 097006 (2006).

[9] M. Ortolani et al., Phys. Rev. Lett. 97, 097002 (2006).

[10] L. Boeri, J. Kortus, O.K. Andersen, Phys. Rev. Lett. 93, 237002 (2004).

[11] K.W. Lee and W.E. Pickett, Phys. Rev. Lett. 93, 237003 (2004).

[12] Y. Ma et al., Phys. Rev. B 72, 014306 (2005).

[13] X. Blase, Ch. Adessi, and D. Connétable, Phys. Rev. Lett. 93, 237004 (2004).

[14] H. J. Xiang et al., Phys. Rev. B 70, 212504 (2004).

[15] F. Giustino, M.L. Cohen, S.G. Louie, Phys. Rev. Lett. (in press).

[16] S. Baroni, A. Dal Corso, S. de Gironcoli, and P. Giannozzi, http://www.pwscf.org.

[17] J. P. Perdew et al. Phys. Rev. Lett. 77, 3865 (1996).

[18] A Fourier-transform technique is used to extrapolate the dynamical matrix on arbitrary q-points (see Ref. 16).

[19] For example, see: M. Chandrasekhar, H.R. Chandrasekhar, M. Grimsditch, M. Cardona, Phys. Rev. B 22, 4825 (1980); C.P. Herrero and M. Stutzmann, Phys. Rev. B 38, 12668 (1988).

[20] Philip B. Allen, Phys. Rev. B 6, 2577 (1972).

[21] Such a technique has been shown to be very efficient in the case of nanotubes. See: D. Connétable et al., Phys. Rev. Lett. 94, 015503 (2005).

[22] Without tetrahedron interpolation, a slightly different $\lambda=0.28$ value was found in Ref. 1 for B:Si.

[23] K.-W. Lee and W.E. Pickett, Phys. Rev. B 73, 075105 (2006).

[24] J.P. Goss and P.R. Briddon, Phys. Rev. B 73, 085204 (2006); E. Bourgeois et al., Phys. Rev. B 74, 094509 (2006). 